\section{ESTADO NUTRICIONAL PRÉ- - CONCECIONAL E AUMENTO PONDERAL NA GRAVIDEZ: ESTUDO DE ACOMPANHAMENTO NA ILHA DO FAIAL}

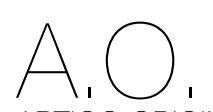

ARTIGO ORIGINAL

1 Unidade de Saúde da ilha do Faial,

Vista Alegre,

9901-853 Horta, Açores,

Portugal

2 Faculdade de Ciências da Nutrição e Alimentação da Universidade do Porto,

Rua do Campo Alegre,

n. ${ }^{\circ} 823$,

4150-180 Porto, Portugal

${ }^{3}$ EPIUnit - Instituto de Saúde Pública da

Universidade do Porto, Rua das Taipas, n. ${ }^{\circ}$ 135, 4050-600 Porto, Portugal

*Endereço para correspondência:

Frederico Viveiros

Rua Alameda Barão de Roches, n. ${ }^{3} 33-\mathrm{A}$,

9900-104 Horta, Açores, Portuga fredericocviveiros@gmail.com

Histórico do artigo:

Recebido a 4 de agosto de 2020 Aceite a 20 de dezembro de 2020

\section{PRECONCEPTION NUTRITIONAL STATUS AND WEIGHT GAIN DURING PREGNANCY: A FOLLOW-UP STUDY IN} THE FAIAL ISLAND

Frederico Viveiros $^{1}$; Rui Poínhos ${ }^{2}$; Cláudia Afonso ${ }^{2,3}$

\section{RESUMO}

É fundamental adequar a evolução ponderal das gestantes, tendo em conta o índice de massa corporal anterior à gravidez e o número de fetos. O aumento de peso inadequado durante a gestação e o estado nutricional das grávidas antes da conceção podem estar associados a riscos para a saúde materna e infantil. A avaliação destes aspetos é fundamental para a definição de estratégias de atuação.

Foi objetivo avaliar o estado nutricional de mulheres do Faial (Açores, Portugal) antes da conceção e o seu aumento de peso durante a gravidez.

Este estudo prospetivo avaliou o estado nutricional anterior à conceção e o aumento de peso durante a gravidez de 34 mulheres com média de idade de 31 anos $(\mathrm{dp}=4)$. Metade da amostra encontrava-se normoponderal antes da conceção e as restantes participantes apresentavam excesso de peso. Verificou-se que $64,7 \%$ da amostra teve um inadequado aumento de peso na gestação, maioritariamente por défice. O aumento de peso durante a gestação não diferiu significativamente entre classes de índice de massa corporal prévio.

Para a melhoria do estado ponderal pré-gestacional recomenda-se um maior foco na educação para a saúde sobre a adoção de estilos de vida saudáveis nas consultas de planeamento familiar. Tendo em conta a elevada inadequação do aumento ponderal na gestação, a evolução ponderal deverá ser monitorizada com maior regularidade, de modo a que o aumento de peso não seja inferior ou superior ao desejável. Uma vez que as recomendações para o aumento de peso durante a gravidez têm em conta o índice de massa corporal prévio, e dada a elevada prevalência de excesso de peso encontrada, esta monitorização deve prolongar-se no período pós-parto. O adequado estado nutricional da mulher antes da conceção e da grávida são fundamentais para otimizar a saúde da própria e do bebé e para diminuir o risco de complicações durante a gestação, pelo que se recomenda a intervenção do nutricionista nestas fases.

\section{PALAVRAS-CHAVE}

Aumento ponderal, Estado nutricional, Gestação, Pré-conceção

ABSTRACT

It is essential to adjust the weight evolution of pregnant women, taking into account the body mass index prior to pregnancy and the number of fetuses. Inadequate weight gain during pregnancy and the nutritional status of pregnant women before conception may be associated with risks to maternal and child health. The evaluation of these aspects is fundamental to the definition of action strategies. The aim was to assess the weight status of women in Faial (Azores, Portugal) before conception and their weight gain during pregnancy.

This prospective study evaluated the nutritional status prior to conception and the weight gain during pregnancy of 34 women with a mean age of 31 years $(S D=4)$. Half of the sample had normal weight before conception and the remaining participants were overweight. It was found that $64.7 \%$ of the sample had an inadequate increase in weight during pregnancy, mostly by deficit. Weight gain during pregnancy did not differ significantly between previous body mass index classes.

To improve the pre-gestational weight status, a greater focus on health education on the adoption of healthy lifestyles in family planning consultations is recommended. In view of the high inadequacy of weight gain during pregnancy, weight evolution should be monitored more regularly, so that weight gain is not less than or greater than desired. Since the recommendations for weight gain during pregnancy take into account the previous body mass index, and given the high prevalence of excess weight found, this monitoring should continue in the postpartum period.

The adequate nutritional status of the woman before conception and the pregnant woman are essential to optimize the health of herself and the baby and to reduce the risk of complications during pregnancy, which is why the nutritionist's intervention in these phases is recommended.

KEYWORDS

Weight gain, Nutritional status, Gestation, Preconception 


\section{INTRODUÇÃO}

A alimentação / nutrição afeta a saúde materna e das gerações futuras. São vários os estudos que reportam um inadequado aporte nutricional antes da conceção e durante a gestação (1-8). Da mesma forma é recomendado adequar a evolução ponderal das gestantes, tendo em conta o índice de massa corporal (IMC) anterior à gravidez e o número de fetos (9). Existe uma elevada percentagem de grávidas com um aumento ponderal desadequado (10-13). Um estudo realizado a nível nacional, com 88 grávidas, aferiu que mais de metade das grávidas normoponderais, $70 \%$ das gestantes com pré-obesidade e $46 \%$ das grávidas com obesidade prévia à conceção tiveram um inadequado aumento ponderal, quer por defeito quer por excesso (12).

O reduzido aumento de peso, para além de aumentar o risco de bebés com menor tamanho para a idade gestacional, também pode estar associado a parto prematuro $(14,15)$, baixo peso à nascença $e$ restrição do crescimento fetal, três situações que podem igualmente estar relacionadas com a obesidade, disfunção metabólica e outras doenças crónicas numa fase mais tardia da vida da criança (16).

Por outro lado, o aumento ponderal excessivo pode originar consequências negativas para o feto, como a macrossomia fetal, independentemente do estado nutricional prévio à conceção (10, 17) e maior tamanho para a idade gestacional (15) mas também afeta a saúde da mãe por aumentar o risco de diabetes gestacional, pré-eclampsia, depressão pós-parto (18) e a necessidade de realizar o parto por cesariana $(15,18)$.

Alterações nos estilos de vida têm demonstrado resultados positivos na redução do ganho de peso na gestação e as intervenções alimentares parecem ser mais eficientes do que intervenções baseadas apenas na atividade física (19). Isto pode ser consequência de uma maior preocupação das grávidas com os seus hábitos alimentares, verificando-se contudo que nem sempre recorrem às fontes de informação mais seguras (20).

Para além do aumento de peso na gestação, o estado ponderal das grávidas antes da conceção também está associado a riscos para a saúde materna e infantil (21-23). Um índice de massa corporal (IMC) pré-gestacional superior ao desejável está associado a maiores níveis de pressão arterial nas crianças desde nascença nascimento (24) e poderá predizer o estado ponderal da mulher no pós-parto (25). O baixo peso antes da gravidez também pode ter consequências negativas, visto que aumenta o risco de parto prematuro e do feto ser pequeno para a idade gestacional (26).

Assim, é importante promover um adequado estado ponderal das grávidas, desejavelmente antes da conceção, para a promoção da saúde das populações (23).

\section{OBJETIVOS}

Este trabalho teve como objetivo avaliar o estado nutricional de mulheres do Faial antes da conceção e o seu aumento ponderal durante a gestação.

\section{METODOLOGIA}

A amostra deste estudo é composta por grávidas que completaram a sua gestação entre maio de 2018 e junho de 2019. Foram critérios de inclusão: idade igual ou superior a dezoito anos, idade gestacional inferior a doze semanas, ausência de comorbilidades (exceto obesidade), ser residente na ilha do Faial e estar inscrita na Unidade de Saúde dessa ilha. As mulheres com história de abortos espontâneos, morte fetal, partos prematuros (inferior a trinta e duas semanas de gestação), tenham sido submetidas a cirurgia bariátrica ou com qualquer condição que condicionasse a participação livre e informada no estudo foram excluídas.

Um total de 48 grávidas foram convidadas a participar no estudo, seis $(12,5 \%)$ das quais recusaram. Foram posteriormente excluídos os dados de seis participantes: três sofreram de aborto espontâneo, uma com parto prematuro (inferior a trinta e duas semanas de gestação) e duas com diagnóstico de diabetes gestacional. Outras duas participantes desistiram do estudo antes do seu término, pelo que foram analisados os dados de 34 mulheres.

Foi aplicado um questionário no final do terceiro trimestre de gestação, sobre informações sociodemográficas (idade quando engravidou, escolaridade, freguesia de residência, estado civil, situação profissional e rendimentos económicos) e dados antropométricos (peso pré-gestacional, altura e aumento ponderal na gravidez). Para o cálculo do IMC pré-gestacional foram considerados os registos presentes no boletim de saúde da grávida e este índice foi classificado segundo os critérios da Organização Mundial da Saúde (27). Para determinar o aumento ponderal durante a gestação foi medido o peso, seguindo os procedimentos da orientação n. ${ }^{\circ}$ 017/2013 da Direção-Geral da Saúde (28), em todas as consultas de acompanhamento e foi avaliada a sua adequação tendo em conta as recomendações do Institute of Medicine (9).

A análise estatística foi realizada no programa IBM SPSS versão 24.0 para Windows. Utilizou-se o teste de Kolmogorov-Smirnov para avaliar a normalidade das variáveis cardinais. A estatística descritiva consistiu no cálculo de frequências absolutas ( $\mathrm{n}$ ) e relativas (\%), médias e desvios-padrão (dp) e de medianas e percentis (P25; P75). O teste de Wilcoxon foi usado para comparar ordens médias de amostras emparelhadas. Rejeitou-se a hipótese nula quando $p<0,05$.

Este estudo foi aprovado pela Comissão de Ética para a Saúde da Unidade de Saúde da llha do Faial, pelo parecer número 4.2018, a 3 de maio de 2018. Todos os participantes deram o seu consentimento informado por escrito e foi-lhes atribuído individualmente um código para assegurar a sua confidencialidade.

\section{RESULTADOS}

A idade média das participantes foi de 31 anos ( $d p=4), 50 \%$ apresentava o ensino superior, $64,7 \%$ estava casada ou em união de facto, a maioria $(91,2 \%)$ estava empregada e $79,4 \%$ considerou os seus rendimentos económicos suficientes para os seus gastos (Tabela 1).

Metade da amostra era normoponderal antes da conceção e as restantes participantes apresentavam excesso de peso. A percentagem de mulheres com pré-obesidade antes da conceção foi ligeiramente superior $(26,5 \%)$ comparativamente às mulheres obesas antes da gravidez (23,5\%).

Verificou-se que o aumento médio de peso na gestação foi de 9,3 kg (dp = 3,6). É de salientar que $64,7 \%$ da amostra não cumpriu as recomendações de aumento de peso na gestação, maioritariamente por défice (défice $(n=15)$ vs. excesso $(n=7))$. Analisando o inadequado aumento ponderal por classes de IMC pré-gestacional verificamos que todas as mulheres normoponderais $(n=11)$ tiveram um reduzido aumento de peso e que $75,0 \%$ das obesas $(n=3)$ tiveram um excessivo aumento ponderal. Relativamente às mulheres com pré-obesidade ( $n=9), 77,8 \%$ tiveram um inadequado aumento ponderal, em que 33,3\% foi por defeito e 44,5\% por excesso (Tabela 2).

Não se verificaram diferenças significativas no IMC pré-gravidez entre as três classes de IMC analisadas, embora se verifique uma tendência para maior IMC pré-gestacional nos casos de inadequação por excesso e o oposto para os de inadequação por defeito (Tabela 3). 
Tabela 1

Caracterização da amostra

\begin{tabular}{|c|c|}
\hline & n (\%) \\
\hline \multicolumn{2}{|l|}{ Idade (anos) quando engravidou } \\
\hline$\leq 29$ & $13(38,2)$ \\
\hline 30 a 35 & $19(55,9)$ \\
\hline$\geq 36$ & $2(5,9)$ \\
\hline \multicolumn{2}{|l|}{ Educação materna } \\
\hline 1. ${ }^{\circ}$ Ciclo incompleto & $2(5,9)$ \\
\hline $2 .^{\circ}$ Ciclo incompleto & $3(8,8)$ \\
\hline 3. ${ }^{\circ}$ Ciclo incompleto & $1(2,9)$ \\
\hline 3. ${ }^{\circ}$ Ciclo completo & $3(8,8)$ \\
\hline Secundário incompleto & $3(8,8)$ \\
\hline Secundário completo & $5(14,7)$ \\
\hline Superior & $17(50,0)$ \\
\hline \multicolumn{2}{|l|}{ Freguesia de residência } \\
\hline Angústias & $4(11,8)$ \\
\hline Capelo & $3(8,8)$ \\
\hline Castelo Branco & $3(8,8)$ \\
\hline Cedros & $2(5,9)$ \\
\hline Conceição & $1(2,9)$ \\
\hline Feteira & $6(17,6)$ \\
\hline Flamengos & $8(23,5)$ \\
\hline Matriz & $2(5,9)$ \\
\hline Pedro Miguel & $1(2,9)$ \\
\hline Praia do Almoxarife & $2(5,9)$ \\
\hline Ribeirinha & $1(2,9)$ \\
\hline Salão & $1(2,9)$ \\
\hline \multicolumn{2}{|l|}{ Estado civil } \\
\hline Solteira & $11(32,4)$ \\
\hline Casada ou em união de facto & $22(64,7)$ \\
\hline Divorciada ou separada & $1(2,9)$ \\
\hline \multicolumn{2}{|l|}{ Situação profissional } \\
\hline Desempregada & $2(5,9)$ \\
\hline Empregada & $31(91,2)$ \\
\hline Doméstica & $1(2,9)$ \\
\hline \multicolumn{2}{|l|}{ IMC pré-gestacional (kg/m²) } \\
\hline$[18,5 ; 25,0[$ & $17(50,0)$ \\
\hline$[25,0 ; 30,0[$ & $9(26,5)$ \\
\hline$\geq 30,0$ & $8(23,5)$ \\
\hline \multicolumn{2}{|l|}{ Número de filhos } \\
\hline 0 & $20(58,8)$ \\
\hline 1 & $12(35,3)$ \\
\hline 2 & $1(2,9)$ \\
\hline 3 & $1(2,9)$ \\
\hline \multicolumn{2}{|l|}{ Patologias } \\
\hline Síndrome vertiginoso & $1(2,9)$ \\
\hline Sinusite e psoríase & $1(2,9)$ \\
\hline Rinite alérgica & $1(2,9)$ \\
\hline Escoliose & $1(2,9)$ \\
\hline Ovários poliquísticos & $1(2,9)$ \\
\hline \multicolumn{2}{|l|}{ Complicações reportadas } \\
\hline Náuseas e/ou vómitos & $17(50,0)$ \\
\hline Descolamento da placenta & $2(5,9)$ \\
\hline Anemia & $3(8,8)$ \\
\hline Obstipação & $3(8,8)$ \\
\hline Prática de atividade física & $21(61,8)$ \\
\hline Caminhada & $17(50,0)$ \\
\hline
\end{tabular}

IMC: Índice de Massa Corporal
Tabela 2

Aumento ponderal total e por classes de Índice de Massa Corporal

\begin{tabular}{|c|c|c|c|c|}
\hline \multirow[b]{3}{*}{ AUMENTO PONDERAL MÉDIO (KG) } & \multicolumn{4}{|c|}{ IMC PRÉ-GESTACIONAL (KG/M²) } \\
\hline & $\mathrm{n}=34$ & {$[18,5 ; 25,0[$} & {$[25,0 ; 30,0[$} & $\geq 30,0$ \\
\hline & $9,3(\mathrm{dp} 3,6)$ & $9,6(d p 3,6)$ & $9,8$ (dp 3,5$)$ & 8,3 (dp 3,9 ) \\
\hline Inadequado por defeito $\left[\mathrm{n}\left(\%^{*}\right)\right]$ & $15(44,1)$ & $11(64,7)$ & $3(33,3)$ & $1(12,5)$ \\
\hline Adequado [n $\left.\left(\%{ }^{\star}\right)\right]$ & $12(35,3)$ & $6(35,3)$ & $2(22,2)$ & $4(50,0)$ \\
\hline Inadequado por excesso [n (\%*)] & $7(20,6)$ & $0(0,0)$ & $4(44,5)$ & $3(37,5)$ \\
\hline
\end{tabular}

IMC: Índice de Massa Corporal

Tabela 3

Índice de Massa Corporal pré-gestacional segundo a classificação do aumento de peso

\begin{tabular}{|c|c|c|c|}
\hline \multirow[b]{2}{*}{ AUMENTO DE PESO NA GRAVIDEZ } & \multicolumn{2}{|c|}{ IMC PRÉ-GESTACIONAL (KG/M²) } & \multirow{2}{*}{ p } \\
\hline & MEDIANA & P25; P75 & \\
\hline Inadequado por defeito [n $\left.\left(\%^{\star}\right)\right]$ & 23,3 & 21,$0 ; 26,3$ & \multirow{3}{*}{0,086} \\
\hline Adequado $\left[\mathrm{n}\left(\%^{*}\right)\right]$ & 24,5 & 21,$2 ; 33,7$ & \\
\hline Inadequado por excesso $\left[\mathrm{n}\left(\%^{\star}\right)\right]$ & 28,7 & 25,$1 ; 31,1$ & \\
\hline
\end{tabular}

IMC: Índice de Massa Corporal

\section{DISCUSSÃO DOS RESULTADOS}

São vários os estudos que apresentam uma proporção de mulheres normoponderais ou com pré-obesidade antes da gravidez próxima da encontrada no presente estudo $(5,8,17,20,29)$, mas a percentagem de mulheres obesas neste mesmo período foi superior comparativamente com outros estudos (1, 2, 8, 17, 20, 29, 30).

Tendo em conta a elevada percentagem de mulheres com excesso de peso antes da conceção recomenda-se um maior foco na educação para a saúde sobre a adoção de estilos de vida saudáveis, nomeadamente prática regular de atividade física e melhoria dos hábitos alimentares, a realizar nas consultas de planeamento familiar. Considerando o carácter preventivo dos cuidados de saúde primários, deve ser feita, sempre que possível, a referenciação ao nutricionista nos casos em que se verifique esta condição.

A inadequação do aumento de peso das participantes que eram normoponderais antes da conceção foi superior à encontrada em vários estudos (12, 31, 32). Nas mulheres que antes da gravidez apresentavam pré-obesidade há estudos que apresentam valores percentuais de inadequação inferiores $(12,31)$ e outros superiores (32, 33). O aumento ponderal na gravidez das participantes com obesidade antes da gestação foi semelhante aos resultados do estudo de Gomes et al. (12), mas outros estudos apontam para percentagens superiores (31-33). Não tendo sido objetivo do presente estudo explorar os motivos conducentes à (in)adequação do aumento ponderal, sugere-se que em trabalhos futuros tal seja avaliado, nomeadamente distinguindo fatores intencionais de não intencionais.

Tendo em conta a reduzida adequação do aumento ponderal na gestação (35,3\%), consideramos que a evolução ponderal deverá ser monitorizada com maior frequência, de modo facilitar o cumprimento das recomendações e visto que estas têm em conta o IMC prévio, e dada a elevada prevalência de excesso de peso encontrada, esta monitorização deve prolongar-se no período pós-parto.

Este trabalho apresenta limitações, nomeadamente ter sido foi realizado em mulheres com um elevado nível de literacia e que residem apenas na ilha do Faial, pelo que os seus resultados não devem ser extrapolados para a população em geral. Para além disso, os valores apresentados podem estar subestimados possivelmente consequência da metodologia utilizada, visto que a última pesagem foi feita antes do final da gestação. 
Foi avaliada a mesma amostra antes da conceção e na gestação, o que minimizou o viés associado às características das participantes. Salienta-se que este é o primeiro estudo, sobre esta temática, realizado na Região Autónoma dos Açores e apenas o segundo feito a nível nacional, que analisa simultaneamente o estado nutricional antes da conceção e a evolução ponderal durante a gravidez, o que reforça a sua pertinência.

\section{CONCLUSÕES}

A elevada prevalência de mulheres que tinham excesso de peso ao engravidar revela a importância da educação para a saúde antes da conceção. A reduzida adequação do aumento de peso na gestação espelham a necessidade de elaborar recomendações mais adequadas a esta população, nomeadamente as que apresentam excesso de peso porque possivelmente não necessitam de aumentar tanto o seu peso. A modificação dos hábitos alimentares tem efeitos positivos que se prolongam ao longo do ciclo de vida da mulher, da criança e de todo o agregado familiar, por isso é essencial a intervenção do nutricionista.

\section{REFERÊNCIAS BIBLIOGRÁFICAS}

1. Dubois L, Diasparra M, Bedard B, Colapinto CK, Fontaine-Bisson B, Morisset AS, et al. Adequacy of nutritional intake from food and supplements in a cohort of pregnant women in Quebec, Canada: the 3D Cohort Study (Design, Develop, Discover). Am J Clin Nutr. 2017;106(2):541-8.

2. Dubois L, Diasparra M, Bedard B, Colapinto CK, Fontaine-Bisson B, Tremblay RE, et al. Adequacy of nutritional intake during pregnancy in relation to prepregnancy BMI: results from the 3D Cohort Study. Br J Nutr. 2018;120(3):335-44.

3. Goletzke J, Buyken AE, Louie JC, Moses RG, Brand-Miller JC. Dietary micronutrient intake during pregnancy is a function of carbohydrate quality. Am J Clin Nutr. 2015;102(3):626-32.

4. Gomes CB, Malta MB, Corrente JE, Benicio MH, Carvalhaes MA. High prevalence of inadequate calcium and vitamin D dietary intake in two cohorts of pregnant women. Cad Saude Publica. 2016;32(12):e00127815.

5. Groth SW, Stewart PA, Ossip DJ, Block RC, Wixom N, Fernandez ID. Micronutrient intake is inadequate for a sample of pregnant african-american women. J Acad Nutr Diet. 2017;117(4):589-98.

6. Harika R, Faber M, Samuel F, Kimiywe J, Mulugeta A, Eilander A. Micronutrient Status and Dietary Intake of Iron, Vitamin A, lodine, Folate and Zinc in Women of Reproductive Age and Pregnant Women in Ethiopia, Kenya, Nigeria and South Africa: A Systematic Review of Data from 2005 to 2015. Nutrients. 2017;9(10).

7. Hatzopoulou K, Filis V, Grammatikopoulou MG, Kotzamanidis C, Tsigga M. Greek pregnant women demonstrate inadequate micronutrient intake despite supplement use. J Diet Suppl. 2014;11(2):155-65.

8. Pinto E, Barros H, dos Santos Silva I. Dietary intake and nutritional adequacy prior to conception and during pregnancy: a follow-up study in the north of Portugal. Public Health Nutr. 2009;12(7):922-31.

9. Rasmussen KM, Yaktine AL, editors. Weight Gain During Pregnancy: Reexamining the Guidelines. The National Academies Collection: Reports funded by National Institutes of Health. Washington (DC)2009.

10. Deputy NP, Sharma AJ, Kim SY. Gestational Weight Gain - United States, 2012 and 2013. MMWR Morb Mortal Wkly Rep. 2015;64(43):1215-20.

11. Deputy NP, Sharma AJ, Kim SY, Hinkle SN. Prevalence and characteristics associated with gestational weight gain adequacy. Obstet Gynecol. 2015;125(4):773-81.

12. Gomes E SA, Campos R. Obesidade e gravidez: conhecer para atuar precocemente? A realidade numa unidade de saúde familiar. Rev Port Endocrinol Diabetes Metab. 2013; 8(1):16-20.

13. Zozzaro-Smith PE, Bacak S, Conway C, Park J, Glantz JC, Thornburg LL. Association Between Obesity During Pregnancy and the Adequacy of Prenatal Care. Matern Child Health J. 2016;20(1):158-63.

14. Chung JG, Taylor RS, Thompson JM, Anderson NH, Dekker GA, Kenny LC, et al. Gestational weight gain and adverse pregnancy outcomes in a nulliparous cohort. Eur
J Obstet Gynecol Reprod Biol. 2013;167(2):149-53.

15. Goldstein RF, Abell SK, Ranasinha S, Misso M, Boyle JA, Black MH, et al. Association of Gestational Weight Gain With Maternal and Infant Outcomes: A Systematic Review and Meta-analysis. JAMA. 2017;317(21):2207-25.

16. Godfrey KM, Gluckman PD, Hanson MA. Developmental origins of metabolic disease: life course and intergenerational perspectives. Trends Endocrinol Metab. 2010;21(4):199-205.

17. Dzakpasu S, Fahey J, Kirby RS, Tough SC, Chalmers B, Heaman MI, et al. Contribution of prepregnancy body mass index and gestational weight gain to adverse neonatal outcomes: population attributable fractions for Canada. BMC Pregnancy Childbirth. 2015;15:21.

18. Marchi J, Berg M, Dencker A, Olander EK, Begley C. Risks associated with obesity in pregnancy, for the mother and baby: a systematic review of reviews. Obes Rev. 2015;16(8):621-38.

19. Thangaratinam S, Rogozinska E, Jolly K, Glinkowski S, Roseboom T, Tomlinson JW, et al. Effects of interventions in pregnancy on maternal weight and obstetric outcomes: meta-analysis of randomised evidence. BMJ. 2012;344:e2088.

20. Aktac S, Sabuncular G, Kargin D, Gunes FE. Evaluation of Nutrition Knowledge of Pregnant Women before and after Nutrition Education according to Sociodemographic Characteristics. Ecol Food Nutr. 2018;57(6):441-55.

21. Dean SV, Lassi ZS, Imam AM, Bhutta ZA. Preconception care: nutritional risks and interventions. Reprod Health. 2014;11 Suppl 3:S3.

22. Grieger JA, Grzeskowiak LE, Clifton VL. Preconception dietary patterns in human pregnancies are associated with preterm delivery. J Nutr. 2014;144(7):1075-80.

23. LifeCycle Project-Maternal O, Childhood Outcomes Study G, Voerman E, Santos S, Inskip H, Amiano P, et al. Association of Gestational Weight Gain With Adverse Maternal and Infant Outcomes. JAMA. 2019;321(17):1702-15.

24. Jansen MAC, Dalmeijer GW, Saldi SR, Grobbee DE, Baharuddin M, Uiterwaal CS, et al. Pre-pregnancy parental BMI and offspring blood pressure in infancy. Eur J Prev Cardiol. 2019;26(15):1581-90.

25. Kirkegaard H, Stovring H, Rasmussen KM, Abrams B, Sorensen TI, Nohr EA. How do pregnancy-related weight changes and breastfeeding relate to maternal weight and $\mathrm{BMI}$-adjusted waist circumference $7 \mathrm{y}$ after delivery? Results from a path analysis. Am J Clin Nutr. 2014;99(2):312-9.

26. Fujiwara K, Aoki S, Kurasawa K, Okuda M, Takahashi T, Hirahara F. Associations of maternal pre-pregnancy underweight with small-for-gestational-age and spontaneous preterm birth, and optimal gestational weight gain in Japanese women. J Obstet Gynaecol Res. 2014;40(4):988-94.

27. World Health Organization. Physical status: the use and interpretation of anthropometry. Report of a WHO Expert Committee. World Health Organ Tech Rep Ser. 1995; 854:1-452.

28. Direção-Geral da Saúde. Orientação n. ${ }^{\circ}$ 017/2013: Avaliação Antropométrica no Adulto. 2013.

29. Abu-Saad K, Shahar DR, Fraser D, Vardi H, Friger M, Bolotin A, et al. Adequacy of usual dietary intake and nutritional status among pregnant women in the context of nutrition transition: the DEPOSIT Study. Br J Nutr. 2012;108(10):1874-83.

30. Chen LW, Tint MT, Fortier MV, Aris IM, Bernard JY, Colega M, et al. Maternal Macronutrient Intake during Pregnancy Is Associated with Neonatal Abdominal Adiposity: The Growing Up in Singapore Towards healthy Outcomes (GUSTO) Study. J Nutr. 2016;146(8):1571-9.

31. Subhan FB, Shulman L, Yuan Y, McCargar LJ, Kong L, Bell RC, et al. Association of pre-pregnancy BMl and gestational weight gain with fat mass distribution and accretion during pregnancy and early postpartum: a prospective study of Albertan women. BMJ Open. 2019;9(7):e026908.

32. Tarqui-Mamani C, Sanabria-Rojas H, Portugal-Benavides WJ, Garcia JC, CastroGaray W, Escalante-Lazo R, et al. [Effectiveness of mobile technology and weight gain in pregnant women in Callao, Peru]. Rev Salud Publica (Bogota). 2018;20(1):67-72. 33 Drehmer M, Duncan BB, Kac G, Schmidt MI. Association of second and third trimester weight gain in pregnancy with maternal and fetal outcomes. PLoS One. 2013;8(1):e54704. 